\title{
Dispositifs d'articulation entre espaces physique et virtuel pour accéder à l'offre de presse
}

Articulating Physical and Virtual Spaces for Access to News Media Distribution

Cécile Payeur et Manuel Zacklad

\section{OpenEdition}

Journals

Édition électronique

URL : http://journals.openedition.org/edc/446

DOI : 10.4000 /edc.446

ISSN : 2101-0366

Éditeur

Université de Lille

Édition imprimée

Date de publication : 1 octobre 2007

Pagination : 39-53

ISBN : 978-2-9514961-9-4

ISSN : 1270-6841

Référence électronique

Cécile Payeur et Manuel Zacklad, « Dispositifs d'articulation entre espaces physique et virtuel pour accéder à l'offre de presse », Études de communication [En ligne], 30 | 2007, mis en ligne le 01 octobre 2009, consulté le 21 décembre 2020. URL : http://journals.openedition.org/edc/446 ; DOI : https:// doi.org/10.4000/edc.446

Ce document a été généré automatiquement le 21 décembre 2020.

(ㄷ) Tous droits réservés 


\title{
Dispositifs d'articulation entre espaces physique et virtuel pour accéder à l'offre de presse
}

\author{
Articulating Physical and Virtual Spaces for Access to News Media Distribution
}

\author{
Cécile Payeur et Manuel Zacklad
}

\section{Introduction}

1 Avec le développement du numérique, les modalités de distribution de la presse connaissent de profondes mutations. Le kiosque traditionnel, qui était aussi un espace de proximité physique, de rencontre, et d'interactions commerciales et sociales, est de plus en plus concurrencé par les kiosques numériques ou virtuels, assurant la distribution des journaux de presse papier et numérique via des espaces marchands sur Internet. Le «même » document presse est désormais accessible à la fois depuis des points d'accès physiques et virtuels. C'est l'intersection de ces deux espaces et la manière de passer de l'un à l'autre qui constitue l'objet de notre étude. Nous nous intéressons aux points de connexion documentaires entre les supports papier et numérique, entre les espaces physique et virtuel. Il s'agit d'envisager la question des interfaces physique/numérique comme autant de portes d'accès, d'ouvertures possibles vers des contenus actualisés qui peuvent avoir différents supports. Loin de s'opposer, ces deux espaces se complètent en effet, et leur imbrication est à inventer. La question que nous nous posons est celle des leviers d'action possibles pour passer d'un espace à un autre, et d'un support à un autre. À travers l'analyse de deux stades d'évolution d'un même projet global d'innovation, notre étude de cas se fonde sur une double expérimentation: l'implantation d'une borne numérique dans un kiosque de presse en région parisienne et celle d'un portail consommateur destiné aux lecteurs de presse sur Internet - dispositifs dont nous avons mené la conception, la mise en place et l'évaluation de septembre 2005 à janvier 2007. Ces deux objets techniques physiques constituent dans leur matérialité des espaces de 
consultation de documents numériques particuliers, que nous appellerons ici documents pivots (catalogues, moteurs de recherche...), et qui contribuent à l'hybridation de l'espace du kiosque de presse en donnant accès soit aux journaux papiers, soit aux journaux numériques. Plus particulièrement, ils nous permettent de dégager trois niveaux d'analyse: le niveau de l'espace physique (le kiosque), le niveau de l'espace numérique dans l'espace physique (la borne interactive) et le niveau de l'espace numérique chez l'usager (le portail Internet). Au-delà de la question de la gestion d'un volume d'informations sans cesse croissant et mouvant, la question des documents pivots renvoie à celle de la pertinence des dispositifs documentaires de médiatisation et de leurs conjonctions possibles dans des espaces publics et privés désormais reliés.

\section{Du document à l'espace}

\section{La matérialité du document}

2 Parler de "mondes virtuels", de "dématérialisation", conduit à penser que la matérialité du document pourrait disparaître. Ce n'est évidemment pas le cas, même si, « la majeure partie de sa réalité concrète demeure souterraine, dissimulée à l'usager au coeur inaccessible d'une machine » (Leleu-Merviel, 2004). La dissimulation de sa matérialité, peutêtre d'une autre nature, nourrit en outre des interrogations sur la valeur ou la validité des documents utilisés dans toutes sortes de transactions dès lors que sont mises en route des procédures de " dématérialisation »; au minimum, elle introduit de facto une coupure entre les acteurs qui accèdent à l'inaccessible et les autres. Il convient donc de réintroduire la matérialité des documents presse numériques en prenant en compte deux points. S. Leleu-Merviel estime qu'un document numérique est, d'une part, «dynamique » et, d'autre part, «collaboratif et partageable» (p. 18). L'auteur collectif Roger T. Pédauque, qui propose une décomposition du document selon trois dimensions distinctes - la forme, le signe et le médium - explicite la dimension de la forme comme étant l'ensemble des « approches qui analysent le document comme un objet, matériel ou immatériel et qui en étudient la structure pour mieux l'analyser, l'utiliser ou le manipuler» (Pédauque, 2003). Cette notion traduit clairement la matérialité du document, ancré dans un support lui-même plongé dans des environnements spatiaux dont la diversité dépend de sa circulation dans ses «états » numérique et papier (imprimé). Pour notre part, dans le cadre conceptuel des Documents pour l'Action (DopA) (Zacklad, 2004), nous visons à intégrer la notion d'objet physique dans la théorie du document tout en insistant sur la dimension collective de l'activité rédactionnelle. Cela permet d'analyser les documents comme relevant de processus de communication pour partie différés, au sens des processus asynchrones décrits dans le champ du CSCW (Computer Supported Cooperative Work ou travail collaboratif assisté par ordinateur), entre des producteurs et des récepteurs liés par des intérêts communs, la matérialité du document apparaît sous l'angle du support : 


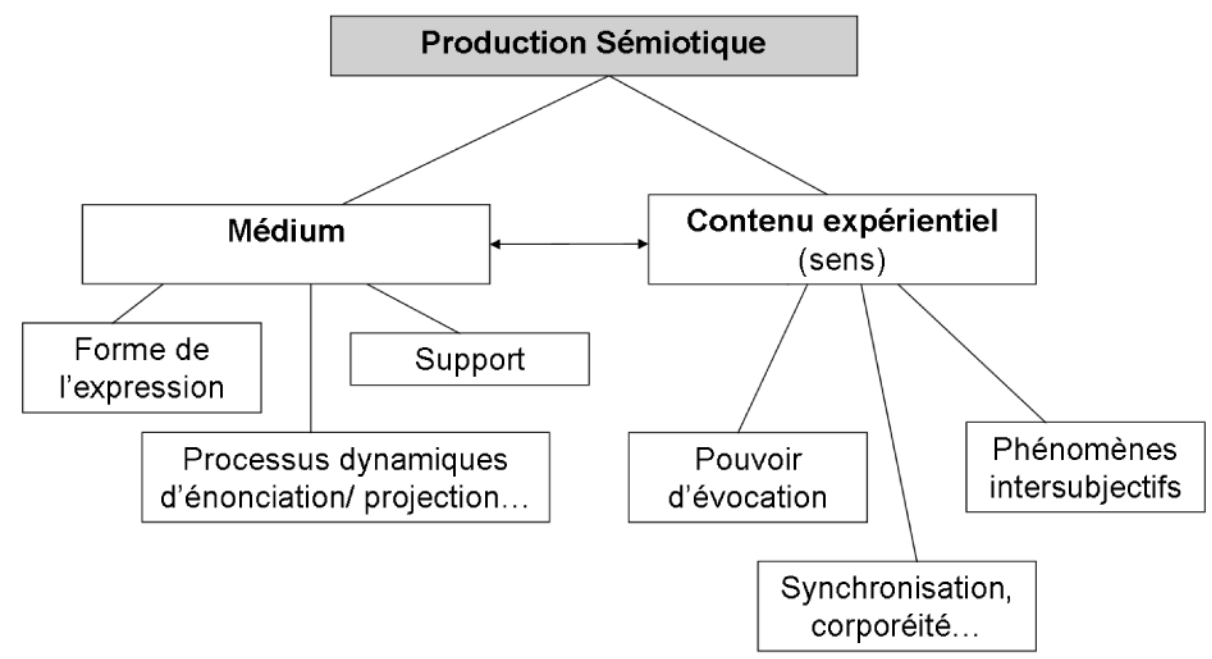

Figure 1 : Analyse de la production sémiotique, adapté de (Zacklad 2007).

La décomposition d'un artefact, vu comme un phénomène transactionnel, amène en effet à établir une distinction entre, d'une part, le plan de la transformation des caractéristiques du médium, et, d'autre part, celui de la transformation du contenu expérientiel des personnes. Une production sémiotique, qui peut être considérée comme un artefact particulier à dominante sémiotique, est donc envisagée selon chacun de ces plans en tant qu'association d'un médium et d'un contenu expérientiel. Le médium se décompose lui-même en une forme d'expression (récit oral, texte écrit, film) et un type de support (présence du narrateur ou enregistrement magnéto pour le récit oral, papier ou électronique pour le texte, DVD ou VHS pour le film, etc.). D'où cette double question: quels sont les types d'espaces sur lesquels ouvre le document? Quelle représentation adopter pour exprimer le lien existant entre le document et l'espace, voire les espaces, qu'il ouvre et où il se déploie?

\section{Le modèle PANUD, un cadre théorique pour innover}

4 Pour apporter quelques éléments de réponse à ces questions, nous avons construit le modèle PANUD (modèle hybride PApier-NUmérique d'édition Diffusion [Payeur \& Zacklad, 2006]). Notre objectif était alors de proposer une représentation du cheminement du document jusqu'au espaces de lecture et de construire un cadre théorique générique destiné à ouvrir des pistes contingentes d'innovations techniques. La construction du modèle PANUD repose sur la base d'un paradigme dû à la numérisation des supports appuyé sur un double constat : tout d'abord, le document subit, à l'heure du numérique, des transformations éditoriales successives, ou "processus d'éditorialisation " (Peyrelong \& Guyot, 2005), au cours duquel ses différentes formes sont manipulées par divers acteurs. Ensuite, la distribution actuelle du document s'effectue sur plusieurs supports et par l'intermédiaire de plusieurs canaux. En ce sens, le modèle PANUD tente de rendre compte non seulement de la variété des modes de composition, de distribution, mais aussi de la variété des modes d'accès aux « documents d'actualité ». A notre sens, le «document d'actualité » le plus représentatif reste le journal, au sens générique du 
terme, c'est-à-dire regroupant à la fois les notions de journal et de magazine. Voici la version générique du modèle PANUD :

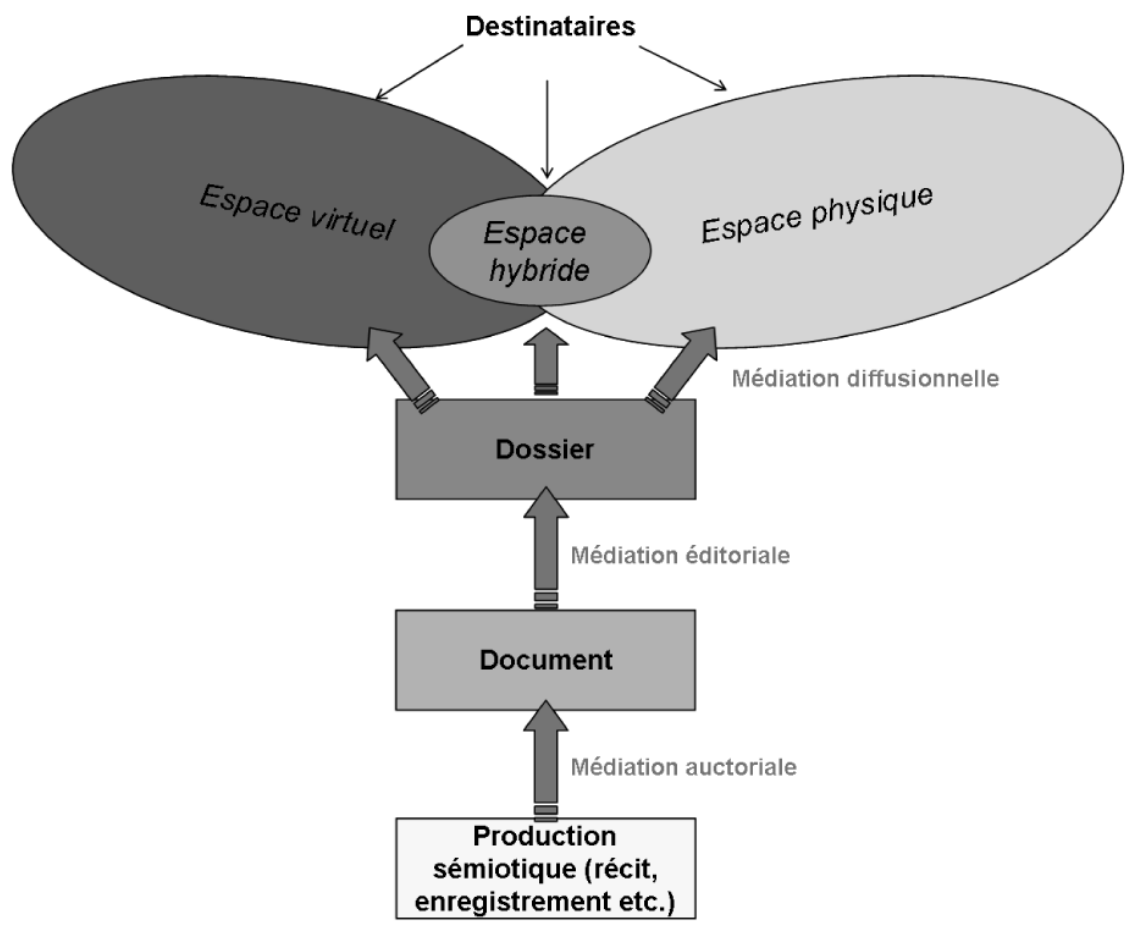

Figure 2 : Modèle PANUD (1) - version générique d'accès aux « documents d'actualité » (Payeur \& Zacklad, 2006)

5 Ce modèle fonctionne sur une lecture à trois niveaux :

- Le niveau de la production sémiotique d'abord, au sens de la théorie des DoPA évoquée précédemment,

- Le niveau du document, ensuite, au sens de cette même théorie, c'est-à-dire en tant que production sémiotique ayant subi une documentarisation consistant à la fois en une pérennisation du support et en l'ajout d'attributs spécifiques visant à faciliter les pratiques liées à son exploitation ultérieure,

- Le niveau du dossier enfin, c'est-à-dire d'une collection documentaire cohérente, documentarisée, associée à un support unique ou à une structure de lien.

6 Correspondant à ces trois niveaux, une même production sémiotique peut subir des médiations de types différents :

- Une médiation auctoriale, lors du passage de la production sémiotique au document, associant :

- un processus triple d'auctorialité qui a trait au champ d'action de l'auteur qui marque par là même son «autorité $»^{1}$ sur la production sémiotique. Ce processus inclut «la production de documents par un ou plusieurs auteurs, la réception par les lecteurs de l'auteur dans l'œuvre ou le document et enfin la liaison permettant de retrouver auteur et document " (Peyrelong \& Guyot, 2005),

- et un processus de documentarisation, au sens de la théorie des DoPA. 
- Une médiation éditoriale ${ }^{2}$, qui s'effectue lors du passage du document au dossier, et qui peut être décrite comme relevant d'une double trajectoire :

- une trajectoire de recomposition produisant une nouvelle hiérarchisation des éléments constitutifs du document,

- une trajectoire de documentarisation, au sens de la théorie des DoPA.

- Une médiation diffusionnelle, qui marque la réception de la production sémiotique par le destinataire, et qui a pour fonction d'ouvrir un espace d'accès jusqu'au destinataire du contenu. Cette dernière médiation, même si elle transforme moins le document, est le point central du processus car c'est le niveau du lien avec le destinataire final. Ce lien s'effectue au sein d'espaces d'accès différents correspondant à deux types de canaux de distribution :

- Les canaux de distribution des produits physiques, tout d'abord, s'appuient sur des réseaux de transport et s'ouvrent sur des espaces physiques allant des espaces de vente au domicile du destinataire,

- Les canaux du virtuel, ensuite, s'appuient sur les réseaux du numérique et débouchent non seulement sur des espaces virtuels, mais potentiellement aussi sur des espaces que nous qualifierons d'espaces "hybrides", qui sont une articulation des deux espaces précédents, et qui émergent au cœur même des espaces physiques (par exemple, des bornes d'achat de billets à l'entrée des cinémas).

\section{La transition entre les espaces chez un distributeur de presse : application du modèle PANUD au cas de la presse écrite}

7 À partir de la version générique du modèle PANUD, nous avons, dans un second temps, bâti une application du modèle destinée à créer un point d'ancrage théorique pour mener à bien un projet global d'innovation chez un distributeur de presse écrite ${ }^{3}$. Cette entreprise, dans le contexte actuel de mutation de la presse écrite, se trouve en effet confrontée à un nécessaire repositionnement dans son domaine. Elle se pose la question de l'exploitation des potentialités offertes par les TIC pour créer de la valeur. Ce qui nous intéresse, c'est la conception de dispositifs permettant d'acquérir un lien direct numérisé avec le lecteur-client, c'est-à-dire se situant au niveau de la médiation diffusionnelle telle qu'elle a été présentée sur le modèle générique.

Voici ce que pourrait donner une version contingente du modèle PANUD, appliquée au cas de la presse écrite : 


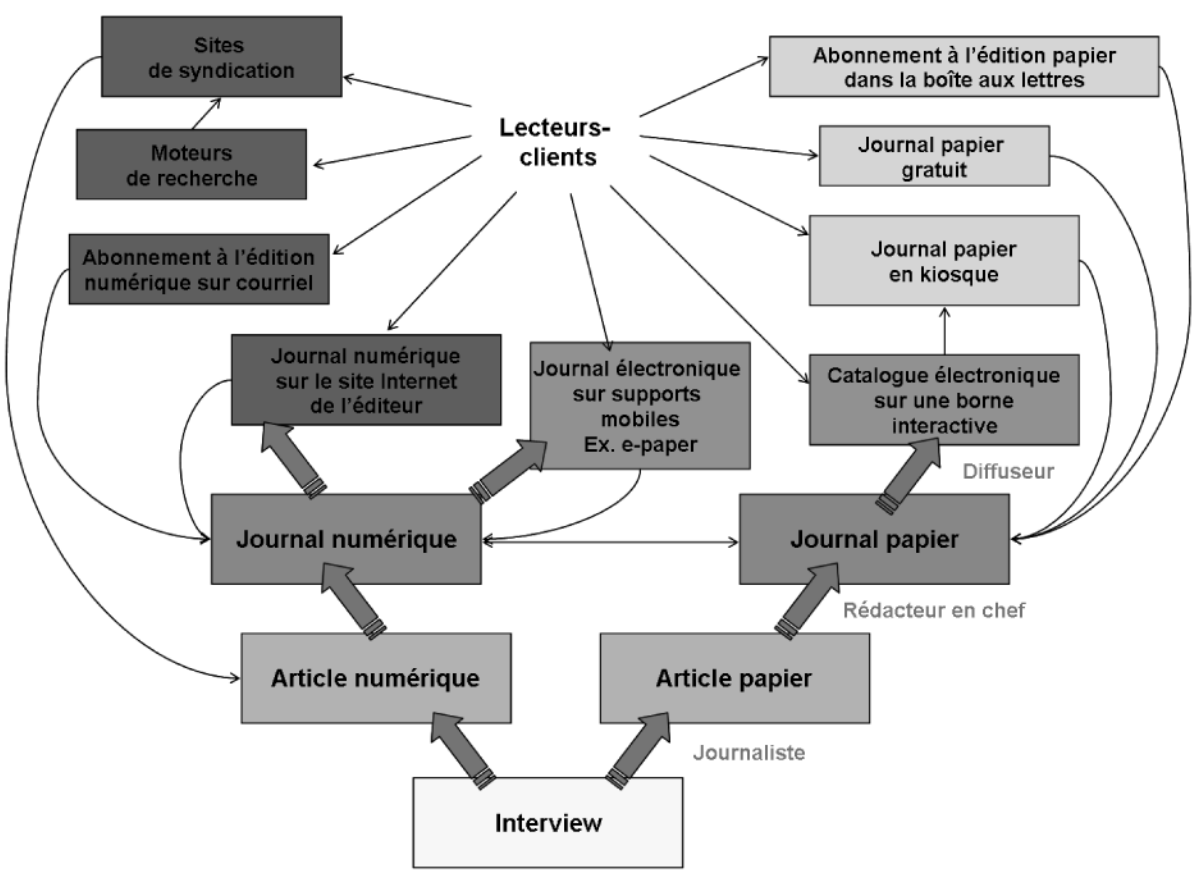

Figure 3 : Modèle PANUD (2) - version contingente d'accès aux journaux dans le secteur de la presse écrite (Payeur \& Zacklad, 2006)

9 On le voit, une même production sémiotique, par exemple une interview, est désormais accessible selon deux niveaux : celui du journal (ou dossier), qui est le mode traditionnel d'accès au contenu journalistique, mais aussi, et de plus en plus fréquemment avec les technologies du numérique, celui de l'article (ou document), en dehors du contexte éditorial du journal. Trois types de médiations peuvent donc potentiellement intervenir :

- Une médiation de type auctoriale au niveau du passage de l'interview à l'article,

- Une médiation de type éditoriale au niveau du passage de l'article au journal (journal papier ou numérique, respect de la maquette, hiérarchisation des articles...).

- Une médiation diffusionnelle finale qui intervient, quant à elle, soit au niveau de l'article, soit au niveau du journal. Elle ouvre, selon les combinaisons adoptées au cours de la ou des médiations précédentes, sur un espace physique, virtuel ou hybride d'accès au contenu.

- D'un côté, dans le cas de la presse écrite, on retrouve le circuit distribution de la presse papier classique, objet physique tangible, et accessible depuis des espaces physiques tangibles. Traditionnellement, le contact avec le lecteur-client se fait au sein du kiosque à journaux, approvisionné par un organisme (le distributeur), et la vente s'effectue par le biais d'une personne physique (le diffuseur). Dans ce type d'espace physique, les problématiques d'accès au document rejoignent très clairement celles du merchandising. Celui-ci a trait à l'appréhension directe du produit physique par le lecteur-client. Cette appréhension passe par l'espace physique du magasin (qui, hormis pour certaines enseignes, n'est actuellement pas normalisé) et se fait par le biais :

- d'une catégorisation par familles correspondant à celles employées par les professionnels de la presse pour classifier les journaux et qui ne répond pas toujours au point de vue du lecteur-client,

- du mobilier, 
- des possibilités de déplacement dans l'espace physique ; il est à noter que certaines zones du magasin, correspondant à des besoins et des types d'achats différents, déterminent des fréquentations diverses et des usages modulés.

- D'un autre côté, on retrouve des circuits du numérique en émergence, accessibles depuis les espaces virtuels que sont les réseaux. Ici, les grandes tendances qui se dessinent sont les suivantes : des journaux en ligne accessibles directement depuis le site des éditeurs de presse, un accès direct au contenu d'un article sorti du contexte du journal par l'intermédiaire de sites de syndication ou l'abonnement à une édition numérique du journal, livrée directement sur le poste informatique du lecteur-client. De plus, on assiste à l'émergence d'espaces que nous qualifierons d'hybrides, dont l'accès repose sur des supports à inventer (le e-paper par exemple).

\section{La mise en place d'une fonction pivot par le biais de deux dispositifs innovants}

Nos travaux nous ont donc amenés à travailler plus particulièrement sur la conception ${ }^{4}$ de deux dispositifs qui utilisent les TIC pour articuler le passage d'un espace à un autre, et, en particulier, le passage de l'espace physique historique, relié par les axes de transport logistiques, aux nouveaux espaces générés par les canaux du numérique. Ils utilisent des documents qui participent de l'hybridation de l'espace et que nous appelons documents pivots.

\section{La borne interactive de recherche d'information, de l'espace hybride vers l'espace physique}

11 Le premier dispositif est une borne interactive de recherche d'information sur le lieu de vente. Il s'agit d'un dispositif positionné au sein de l'espace hybride, permettant d'accéder aux journaux papier par l'intermédiaire d'un catalogue numérique virtuel. La borne est tout d'abord un objet physique, dont la matérialité s'appréhende par un support sur pieds positionné dans le magasin à hauteur des yeux, mais surtout par un écran tactile que le lecteur-client touche pour accéder au contenu. Ce même objet physique permet l'accès à des documents numériques sous la forme d'un catalogue, consultable par familles ou par moteur de recherche. Ce catalogue ne reproduit pas le contenu intégral des journaux papiers présents dans le point de vente, mais utilise un balisage par mots-clés sur des parties de ce même contenu. Le dispositif permet à la fois au lecteur-client de naviguer dans le catalogue des journaux présents dans le point de vente physique, et d'effectuer une recherche par thème sur le contenu du sommaire de ces mêmes journaux. Il a donc pour double fonction de renseigner, mais aussi de permettre au lecteur-client de découvrir les documents papiers présents dans le kiosque. L'objectif est donc d'offrir à un lecteur-client mieux renseigné une bonne accessibilité dans l'offre, et de stimuler la vente des journaux papier. À terme, il est également envisagé d'acheminer dans le magasin les journaux qui pourraient intéresser le lecteur-client et que le diffuseur n'a pas reçu en attribution choisie par l'éditeur.

12 La construction d'une maquette de cette borne interactive nous a permis de simuler la mise en situation réelle de la borne, début 2006, chez un diffuseur de presse en région parisienne. Une soixantaine de personnes ont été ainsi confrontées directement au dispositif et interrogées. L'enquête a été complétée par une approche de type 
ethnographique fondée sur l'observation à la fois directe et filmée des lecteurs-clients fréquentant le magasin, ainsi que du diffuseur de presse. Cette expérimentation a duré près de deux semaines ${ }^{5}$.

\section{Le portail Internet de syndication de contenu, de l'espace virtuel vers l'espace physique}

13 Le portail Internet de syndication de contenu est la déclinaison Internet du dispositif borne. Il permet d'accéder à un catalogue basé sur un accès par familles. Il permet également de rechercher un journal par centre d'intérêt grâce à l'utilisation d'un moteur de recherche. Le contenu des documents pivots est, quant à lui, enrichi des informations relatives aux flux RSS des différents journaux. Ceux-ci permettent d'ajouter de la pertinence à la recherche. Ce dispositif, situé au sein de l'espace virtuel, est conçu dans l'optique d'aller chercher le lecteur-client directement chez lui pour le ramener vers le point de vente physique. Dans ce dessein, il est directement relié à des fonctions de géolocalisation des points de vente (basées notamment sur Google Map), mais aussi à des fonctions de localisation par familles dans les rayons.

Ici, notre démarche concernant l'expérimentation s'est également appuyée sur la mise en situation réelle d'une maquette du dispositif auprès d'une trentaine d'utilisateurs aux profils variés (internes et externes à l'entreprise, plus ou moins familiarisés avec l'usage des nouvelles technologies etc.). Il s'agit d'un premier pas avant l'intégration des fonctionnalités pivots du dispositif à un portail opérationnel déjà développé.

\section{La triple fonction des documents pivots}

$15 \mathrm{Au}$ terme de ces expérimentations, les conclusions principales que nous avons pu tirer la fonction des documents pivots que nous avons mis en place furent de trois ordres.

\section{L'élargissement des points de vue du lecteur-client}

16 Sur la borne, l'élargissement s'effectue grâce à une nouvelle entrée dans le contenu des documents. En effet, les familles proposées par le catalogue ne sont plus uniquement les familles professionnelles que l'on trouvait dans l'espace physique, mais de nouvelles familles construites en fonction des différents points de vue et des centres d'intérêt du lecteur-client. En ce sens, un moteur de recherche par mots-clés vient compléter l'accès par catalogue et permet de rechercher directement tous les journaux qui sont en vente et «qui traitent de... » ou " qui parlent de... ». Elles sont liées aux familles de classifications professionnelles évoquées précédemment par la fonction de localisation des produits dans le magasin.

Sur le portail, l'élargissement des points de vue est sensiblement identique à celui de la borne, avec ceci de particulier, que la mise en réseau par Internet permet d'enrichir le contenu des documents pivots, et d'imaginer à terme des collaborations et des modalités de partage d'information entre utilisateurs. 


\section{Une carte d'aiguillage entre les espaces}

18 Sur la borne, finalement, la borne intervient directement là où les contraintes de l'espace physique ne permettent plus de répondre à la demande du lecteur-client et, ceci, à un double niveau, correspondant à la double entrée du modèle PANUD appliqué à la presse écrite: au niveau du journal tout d'abord (elle permet de souligner les journaux non visibles, de faire découvrir de nouveaux titres, de vérifier que le dernier numéro d'un journal est sorti...) et au niveau de l'article ensuite (c'est par l'entrée par thème que le lecteur-client accède directement au sujet traité par les articles). Spontanément, dans les deux cas, et lorsque le dispositif hybride a répondu à la demande, il y a déplacement du lecteur-client dans l'espace qui se dirige de la borne virtuelle au rayon physique qui se trouve directement à proximité. La transition entre le catalogue virtuel et le journal papier, et donc de l'espace physique à l'espace physique, se fait donc pour ainsi dire naturellement.

Sur le portail, le mécanisme de réponse à la demande est sensiblement le même, mais le déplacement que va devoir effectuer le lecteur-client au sein des espaces est double. Dans ce cas, le dispositif capte le lecteur-client en dehors du point de vente, là où il se trouve (le plus souvent, directement à son domicile). Lorsque le dispositif a répondu à sa demande, il faut tout d'abord lui permettre d'identifier le kiosque le plus proche qui possède le journal en stock grâce à une fonction de géolocalisation. Il faut ensuite lui permettre de trouver le journal dans le point de vente, selon le même processus qu'utilisé précédemment sur la borne. Les documents pivots contenus dans le portail doivent stimuler un déplacement physique entre ces deux espaces: ils doivent faire passer le lecteur-client de l'espace virtuel jusqu'à l'espace physique.

\section{Les artefacts de la prescription ${ }^{6}$}

Sur la borne, qui a d'abord été pensée et conçue pour le lecteur-client, nous avons noté des utilisations spontanées, et, d'une certaine façon, inattendues, du dispositif par le diffuseur de presse. Celui-ci y a trouvé un support qui lui faisait défaut pour conseiller sa clientèle et répondre à certaines questions posées dans des contraintes de temps souvent assez fortes. Ainsi, au cours de notre expérimentation, un lecteur-client ayant vu par l'intermédiaire d'un autre média, la télévision, une annonce pour un magazine consacrant un reportage à la chirurgie esthétique a demandé au diffuseur : «je cherche un magazine féminin où il y a un article sur la chirurgie esthétique ce mois-ci... ». Le diffuseur a pu utiliser la borne pour entrer les mots clés « chirurgie » et " esthétique ». La couverture de tous les journaux qui traitaient de ce sujet à cette date est alors apparue sur l'écran permettant au lecteur-client de reconnaitre le titre recherché : « le voici !». Le diffuseur, le trouvant le titre dans ses rayons a pu proposer le produit physique au client ce qui l'a conduit à une évaluation positive: «merci, sans vous j'aurais raté une vente [...] ! Vous comprenez, l'offre est tellement large que je ne peux pas me souvenir de tout ce qui sort chaque jour et, bien souvent, c'est malheureusement lorsque j'enlève les journaux non vendus des rayons que j'ai le temps de regarder de quels sujets ils traitent... Je manque énormément de temps lors de la mise en place!». Cette utilisation de la borne par le diffuseur a également comme conséquence de lever un des freins à son utilisation par certains clients, qui craignent de voir l'outil « informatique » remplacer le vendeur, comme on a pu le voir sur les bornes de vente de tickets à l'entrée du métro par exemple. 
21 Sur le portail, la fonction de recherche et les manières d'accéder au contenu sont libres. Elles ne peuvent en aucun cas être accompagnées de la présence du diffuseur. La nécessité de renforcer les capacités de prescription autonome du dispositif est donc renforcée. En ce sens, le contenu est enrichi d'informations actualisées et passant par le réseau Internet, informations qui ne se trouvent pas nécessairement sur le point de vente.

\section{Conclusion et perspectives}

L'observation de ces deux expérimentations nous permet de tirer des premières conclusions sur les conditions de passage du document d'un espace à un autre. Tout d'abord, celui-ci impose à la fois le choix d'une sémantique permettant l'adoption du point de vue du destinataire final et une représentation visuelle de ces espaces permettant d'aiguiller un déplacement physique de la personne. En ce sens, les documents pivots constituent pour nous de véritables cartes sémantiques numériques. Celles-ci doivent être également accompagnées d'outils permettant de renforcer la fonction de prescription du dispositif. Ce point est d'autant plus important que l'éloignement physique entre les deux espaces à joindre est grand, comme dans le cas où la consultation sur écran doit induire une visite chez un diffuseur.

Grâce au modèle présenté et aux expérimentations, nous avons tenté de tracer quelques lignes permettant de comprendre l'action des documents pivots sur l'hybridation de l'espace, afin d'en maîtriser leur conception et de trouver les manières de «capter » et "d'orienter" le lecteur-client d'un espace à un autre selon ses besoins. Par-delà les questions inhérentes à la conception de ce type de document, se pose clairement la question de la création de valeur et de son évaluation. La gestion de l'espace fait en effet intervenir différents acteurs et réseaux d'acteurs - c'est la problématique de la distribution - chaque acteur cherchant à capter de la valeur. Il s'agit pour nous d'un des enjeux majeurs liés à la conception des documents pivots, notamment dans leur fonction de prescription. Cette conception dépend en particulier du type de valeur que l'on veut créer. Ce point fera, entre autres, l'objet de la suite de nos investigations.

\section{BIBLIOGRAPHIE}

Benghozi, P. J., (2003), Paris T., " De l'intermédiation à la prescription : le cas de l'audiovisuel », Revue Française de Gestion, vol. 29, n 402, pp. 205-207, janv.-fév. 2003.

Broudoux, E., Bootz, P., Clément, J., Grésillaud, S., Le Crosnier, H., Lux-Pogodalla, V., Réty, J.-H., Rojas, E. et Vidal, G., (2005), Auctorialité : production, réception et publication de documents numériques, in : La redocumentarisation du monde, Pédauque Roger T. (éd.), Toulouse, Cepadues.

Broudoux, E., (2007), Construction de l'autorité informationnelle sur le Web, archive SIC [en ligne], 17 janvier 2007, version 1, disponible à l'adresse suivante : http://archivesic.ccsd.cnrs.fr/ sic_00120710 (consultée le 10 mars 2007). 
Leleu-Merviel, S., (2004), « Effets de la numérisation et de la mise en réseau sur le concept de document », Revue Information - Interaction - Intelligence, vol. 4, n 1, pp. 121-140.

Payeur, C. et Zacklad, M., (2006), Modèle d'accès multi-supports et multi-canaux aux documents d'actualité, CIDE. 9 ; $9^{\mathrm{e}}$ Colloque International sur le Document Électronique, SDN'06 ; $6^{\mathrm{e}}$ Semaine du Document Numérique, Fribourg Suisse, 18-20 Septembre 2006.

Pédauque, R. T., (2003), Document : forme, signe et medium, les re-formulations du numérique, 08 juillet 2003, working paper, archive SIC [en ligne], disponible à l'adresse suivante : "http:// archivesic.ccsd.cnrs.fr/sic_00000511.html" http://archivesic.ccsd.cnrs.fr/sic_00000511.html (consultée le $1^{\mathrm{er}}$ mars 2006).

Peyrelong, M. F. et Guyot, B., (2005), « Quelques résultats pour les sciences de l'information », chapitre du rapport final Action Spécifique « document et organisation », RTP CNRS, 2005.

Zacklad, M., (2007), Processus de documentarisation dans les Documents pour l'Action (DopA). Babel - edit -, Le numérique : impact sur le cycle de vie du document. ENSSIB - février 2007 [en ligne] : http://babel.enssib.fr/document.php?id=727.

\section{NOTES}

1. Pour nous, la médiation auctoriale fait référence à "l'autorité énonciative ", en tant "qu'autorité des auteurs jugés selon leur reconnaissance, la qualité et le nombre de produits publiés, leur notoriété actuelle et passée » (Broudoux, 2007) voir aussi (Broudoux et al., 2005).

2. A ce niveau, la médiation éditoriale fait référence à "l'autorité de contenu», en tant « qu'identification par le genre éditorial » (Broudoux, 2007).

3. L'un des auteurs est responsable d'une entité chargée de l'innovation au sein de cette même entreprise.

4. Nous ne reviendrons pas ici sur la partie pilotage des projets d'innovation, qui ferait l'objet d'une autre étude.

5. Sur ce projet, depuis mars 2007, un prototype est désormais en phase de test élargie sur une dizaine de kiosques et cette nouvelle phase nous permettra de confirmer - ou d'infirmer - les premières conclusions expérimentales tirées de la maquette.

6. La notion de prescription s'entend ici telle que revisitée sous l'angle économie gestion par P.J. Benghozi et présentant l'avantage, notamment par élargissement de la notion d'intermédiation, "de (permettre de) s'intéresser plus spécifiquement aux modalités de décision des consommateurs et aux modes de structuration des relations à la base de la prescription » (Benghozi, 2003).

\section{RÉSUMÉS}

Avec le développement des réseaux du numérique, le document ouvre désormais sur des espaces physiques, mais aussi sur des espaces virtuels en émergence. Questionner la façon dont les documents pivots permettent d'articuler le passage d'un espace à un autre met à jour des mécanismes de transition nouveaux et qu'il s'agit de maîtriser. Nous tenterons d'en appréhender 
les fonctions principales à travers l'expérimentation de deux dispositifs innovants basés sur les TIC dans le cas de la distribution de la presse.

The expansion of digitalization and networks facilitate the dissemination of documents in physical spaces but also in emerging virtual spaces. This paper explores the articulation between physical and virtual spaces thanks to pivotal documents allowing new transitional mechanisms. The paper captures their main functionalities through experimentation with two innovative sociotechnical systems based on information and communication technology (ICT) in the press distribution sector.

\section{INDEX}

Keywords : pivotal document, press, space, design, innovation, physical space, virtual space Mots-clés : document pivot, presse, espace, conception, innovation, espace physique, espace numérique

\section{AUTEURS}

\section{CÉCILE PAYEUR}

Cécile Payeur : Après un diplôme d'ingénieur de l'Université de Technologie de Compiègne et quelques années en entreprise (Berlin), Cécile Payeur effectue une thèse CIFRE en tant que chef de projet innovation dans les domaines de l'ingénierie des connaissances et des sciences de l'information au sein du service marketing d'un distributeur de presse. Cette thèse est dirigée par Manuel Zacklad à l'Université de Technologie de Troyes au sein du laboratoire pluridisciplinaire Tech-CICO (UTT/ICD Tech-CICO FRE CNRS 2848). Elle a déjà présenté plusieurs communications dans des conférences en information-communication et en informatique documentaire. Adresse électronique : cecile.payeur@utt.fr.

\section{MANUEL ZACKLAD}

Manuel Zacklad est professeur des Universités en sciences cognitives et en système d'information et directeur du laboratoire pluridisciplinaire Tech-CICO à l'Université de Technologie de Troyes (UTT/ICD Tech-CICO FRE CNRS 2848). Ses thèmes d'intérêts couvrent l'usage des documents en milieu professionnel (documents pour l'action, processus de documentarisation, annotations...), le CSCW et les communautés d'action, la gestion des connaissances basée sur le cadre théorique de la socio-économie des transactions coopératives et le web socio-sémantique pour la recherche ouverte d'information. Adresse électronique : manuel.zacklad@utt.fr. 